\title{
The antitumor immune responses induced by nanoemulsion- encapsulated MAGE1-HSP70/SEA complex protein vaccine following different administration routes
}

\author{
WEI GE ${ }^{1,2^{*}}$, PEI-ZHEN HU ${ }^{1 *}$, YANG HUANG ${ }^{1}$, XIAO-MING WANG ${ }^{2}$, XIU-MIN ZHANG $^{1}$, \\ YU-JING SUN ${ }^{1}$, ZENG-SHAN LI ${ }^{1}$, SHAO-YAN SI ${ }^{1}$ and YAN-FANG SUI ${ }^{1}$ \\ ${ }^{1}$ Department of Pathology, State Key Laboratory of Cancer Biology; ${ }^{2}$ Department of Geriatrics, \\ Xijing Hospital, Fourth Military Medical University, Xi'an, Shaanxi Province 710032, P.R. China
}

Received March 31, 2009; Accepted May 6, 2009

DOI: 10.3892/or_00000517

\begin{abstract}
Our previous study showed that nanoemulsionencapsulated MAGE1-HSP70/SEA (MHS) complex protein vaccine elicited MAGE-1 specific immune response and antitumor effects against MAGE-1-expressing tumor and nanoemulsion is a useful vehicle with possible important implications for cancer biotherapy. The purpose of this study was to compare the immune responses induced by nanoemulsion-encapsulated MAGE1-HSP70 and SEA as $\mathrm{NE}(\mathrm{MHS})$ vaccine following different administration routes and to find out the new and effective immune routes. Nanoemulsion vaccine was prepared using magnetic ultrasound methods. C57BL/6 mice were immunized with NE(MHS) via po., i.v., s.c. or i.p., besides mice s.c. injected with PBS or NE(-) as control. The cellular immunocompetence was detected by ELISpot assay and LDH release assay. The therapeutic and tumor challenge assay were also examined. The results showed that the immune responses against MAGE-1 expressing murine tumors elicited by NE(MHS) via 4 different routes were approximately similar and were all stronger than that elicited by PBS or NE(-), suggesting that this novel nanoemulsion carrier can exert potent antitumor immunity against antigens encapsulated in it. Especially, the present results indicated that nanoemulsion vaccine adapted to administration via different routes
\end{abstract}

Correspondence to: Dr Xiao-Ming Wang, Department of Geriatrics, Xijing Hospital, Fourth Military Medical University, Xi'an, Shaanxi Province 710032, P.R. China

E-mail: xmwang@fmmu.edu.cn

Dr Yan-Fang Sui, Department of Pathology, State Key Laboratory of Cancer Biology, Xijing Hospital, Fourth Military Medical University, Xi'an, Shaanxi Province 710032, P.R. China

E-mail: suiyanf@fmmu.edu.cn

${ }^{*}$ Contributed equally

Key words: tumor vaccine, nanoemulsion, peroral route including peroral, and may have broader applications in the future.

\section{Introduction}

Tumor vaccines based on tumor-specific antigen (TSA) play an important role in the prevention and therapy of tumors and have been regarded as an attractive method. The melanoma antigen (MAGE) was the first reported example of TSA. MAGE-1 is an important member of MAGE, which is expressed in most tumors but not in normal tissues except the testes and placenta. Moreover, MAGE-1 antigen has been termed as tumor-rejection antigens because tumors expressing these antigens on appropriate human leukocyte antigen (HLA) class I molecules are rejected by host cytotoxic T lymphocytes (CTLs) (1).

Heat shock protein (HSP), as a molecular chaperone, participating in processing and presentation of tumor antigen and plays an important role in eliciting antitumor immunity. Related research has shown that HSP70 could be exploited to enhance the cellular and humoral immune responses against any attached tumor-specific antigens (2).

Staphylococcal enterotoxins A (SEA) is a classical model of superantigens. It forms a complex with MHC class II molecules on antigen-presenting cells, binds to the outside of the antigen binding cleft to stimulate as much as $20 \%$ of the $\mathrm{T}$-cell repertoire via $\mathrm{VB}$-specific elements of the T-cell receptor (3). Both $\mathrm{CD}^{+}$and $\mathrm{CD}^{+} \mathrm{T}$ cells have been shown to proliferate in response to these superantigens (4). This massive activation of $\mathrm{T}$ cells is accompanied by an increased production of cytokines such as interferon- $\gamma(\mathrm{IFN}-\gamma)$.

Related studies (5) indicate that vaccinal approach using nano-delivery system carrying the tumor-specific antigens can be developed to enhance the cellular and humoral immune responses against antigens encapsulated in nanoemulsion. Our previous (unpublished) study showed that nanoemulsion-encapsulated MAGE1-HSP70/SEA complex protein vaccine produces better MAGE-1-specific cellular immune response and antitumor effect, and the best ratio is 100:1, at which ratio the maximal antitumor effect and the minimal toxicity or tolerance be exerted. However, subcutaneous (s.c.) or intraperitoneum (i.p.) injection was the 
standard in our former studies. In this study, C57BL/6 mice were immunized via po., i.v., s.c. or i.p. The goal was to investigate the potential benefit of using W/O nanoemulsion as an alternative carrier of MHS complex protein vaccine, which can be adapted to administration via different routes including po.

\section{Materials and methods}

Animals. C57BL/6 mice (6- to 8-week-old) were obtained from the Laboratory Animal Center of the Fourth Military Medical University (Xi'an, China). Mice were housed in microisolation in a dedicated, pathogen-free facility, and all animal experimentation was conducted in accordance with the declaration of Helsinki or NIH guidelines.

Cell lines. B16 cell line and B16-MAGE-1 cell line (6) were conserved in our laboratory. On the day of tumor challenge, B16-MAGE-1 cells were harvested and finally resuspended in $1 \times P B S$ for injection.

Encapsulation of vaccine. MAGE1-HSP70 fusion protein and SEA was constructed, purified and conserved in our laboratory. Soybean oil, Pluronic 188 and Span-20 were obtained from Sigma (Sigma Chemicals, Saint-Louis, MO, USA). Water was bidistilled. All chemicals and solvents were used without further purification. MHS nanoemulsion was prepared using magnetic ultrasound method. Briefly, $1.0 \mathrm{ml} \mathrm{0.1 \%} \mathrm{(w/w,} 1 \mathrm{mg}$ ) MHS protein (the ratio of MAGE1HSP70 to SEA was $100 \mathrm{~mol}: 1 \mathrm{~mol}$ ) was added to solution containing $18 \%(\mathrm{v} / \mathrm{v})$ Pluronic 188 and $8 \%(\mathrm{v} / \mathrm{v})$ Span-20. Soybean oil $0.6 \mathrm{ml}$ was introduced to this system and mixed, and the oil phase was obtained by adjusting the mixture volume to $2.5 \mathrm{ml}$ with bidistilled water. Afterwards, the oil phase was added dropwise into the $7.5 \mathrm{ml}$ bidistilled water while aqueous phase was stirred under magnetic power (3000 rpm). Then the mixture was put into the vacuum highspeed sheering emulsification device (FM600, Fluko Inc., Germany), sheared with $23000 \mathrm{rpm}$ under $0.7 \mathrm{kpa}$ vacuum pressure for $40 \mathrm{~min}$ at no higher than $80^{\circ} \mathrm{C}$, followed by process with ultrasound generator $(20 \mathrm{kHz}, 75$ watt ColeParmer International Inc., USA) at $0^{\circ} \mathrm{C}, 5 \mathrm{~min}, 3$ times. Finally, we got a half-transparent fluid with the concentration of $100 \mu \mathrm{g} / \mathrm{ml}$ MHS. MAGE1-HSP70 and SEA not encapsulated within nanoemulsion were removed by dialyzing using $90 \mathrm{kDa}$ dialyser and were quantitated by high performance liquid chromatography (HPLC). The encapsulation efficiency was determined by the following formula: Encapsulation efficiency $=($ Total drug concentration-Free drug concentration $) /$ Total drug concentration $\times 100 \%$. The morphology of ADM-FDNG was evaluated by transmission electron microscopy (TEM, Hitachi S-520, Tokyo, Japan) observations. Five hundred nanoemulsions were examined for average size and size distribution. NE(MHS) was stored at $4^{\circ} \mathrm{C}$ before use.

Immunization regime. Thirty-six C57BL/6 mice were divided into 6 groups: po. NE(MHS), i.v. NE(MHS), s.c. NE(MHS), i.p. NE(MHS), PBS and NE(-) group. NE(MHS) (150 pmol/ $150 \mu 1$ /mouse) was administrated via po., i.v., s.c. or i.p. route respectively in the 4 groups. Mouse was s.c. injected with
$150 \mu 1$ PBS or NE(-) as control. Each mouse was immunized every 10 days, three times. The splenocytes were harvested and pooled 10 days after the boost.

IFN- $\gamma$ enzyme-linked immunosorbent spot (ELISpot) assay. Mouse IFN- $\gamma$ ELISpot assay was performed in PVDFbottomed 96-well plates (Millipore, Bedford, MA, USA) by using a murine IFN- $\gamma$ ELISpot kit (Diaclone, Besancone, France) according to the manufacturer's instructions with minor modifications. Briefly, plates were coated overnight at $4^{\circ} \mathrm{C}$ with anti-IFN- $\gamma$ capture antibody and washed three times with PBST (PBS+0.1\% Tween-20). Plates were blocked for $2 \mathrm{~h}$ with $2 \%$ skimmed dry milk. Splenocytes $\left(5 \times 10^{5}\right.$ cells/ well) were then added together with the indicated number of lethally irradiated (10000 cGy) B 16-MAGE-1 cells (5x104/well respectively) and incubated for $24 \mathrm{~h}$ at $37^{\circ} \mathrm{C}$. Cells were then removed and a biotinylated IFN- $\gamma$ detection antibody was added for $2 \mathrm{~h}$. Free antibody was washed out, and the plates were incubated with streptavidin-alkaline phosphatase for $1 \mathrm{~h}$ at $37^{\circ} \mathrm{C}$, followed by extensive washing with PBST, and with PBS. Spots were visualized by the addition of the alkaline phosphatase substrate BCIP/NBT. The number of dots in each well was counted using a dissection microscope. The number of MAGE-1-specific T-cell precursors in splenocytes was calculated by subtracting the IFN- $\gamma^{+}$spots of splenocytes on B16 stimulating cells from that on B16-MAGE-1 cell.

Cytotoxicity assay. The CytoTox 96 non-radioactive cytotoxicity assay (Promega Inc.) was performed to determine the cytotoxic activity of the splenocytes in mice vaccinated with various proteins against B16-MAGE-1 tumor cells, according to the manufacturer's protocol with minor modification. Briefly, splenocytes of vaccinated mice were cultured in the presence of human IL-2 (40 U/ml) and irradiated B16MAGE-1 cells. After 3 days, B16 and B16-MAGE-1 target cells were plated at $1 \times 10^{4}$ cells/well on 96 -well U-bottomed plates (Costar), then the splenocytes (effector cells) were added in a final volume of $100 \mu 1$ at 1:5, 1:20 and 1:80 ratio, respectively. The plates were incubated for $45 \mathrm{~min}$ in a humidified chamber at $37^{\circ} \mathrm{C}, 5 \% \mathrm{CO}_{2}$, and centrifuged at $500 \mathrm{x}$ g for $5 \mathrm{~min}$. Aliquots $(50 \mu \mathrm{l})$ were transferred from all wells to a fresh 96-well flat-bottom plate, and an equal volume of reconstituted substrate mix was added to each well. The plates were incubated at room temperature for $30 \mathrm{~min}$ and protected from light. Then $50 \mu 1$ stop solution was added, and the absorbance values were measured at $492 \mathrm{~nm}$. The percentage of cytotoxicity for each effector: target cell ratio was calculated from the equation: [A (Experimental) - A (Effector Spontaneous) - A (Target Spontaneous)]x100/[A (Target maximum) - A (Target spontaneous)]. Percentage of MAGE-1-specific lysis was calculated by subtracting the lysis percentage of splenocytes on B16 from that on B16MAGE-1 target cells.

In vivo tumor treatment experiments. Sixty mice (10 per group) were s.c. challenged with B16-MAGE-1 tumor cells ( $1 \times 10^{5}$ cells/mouse, respectively) in the right legs (D0). Seven days later, 40 mice were vaccinated with $150 \mathrm{pmol} /$ $150 \mu \mathrm{l} /$ mouse NE(MHS) by po., i.v., s.c. or i.p. route respec- 


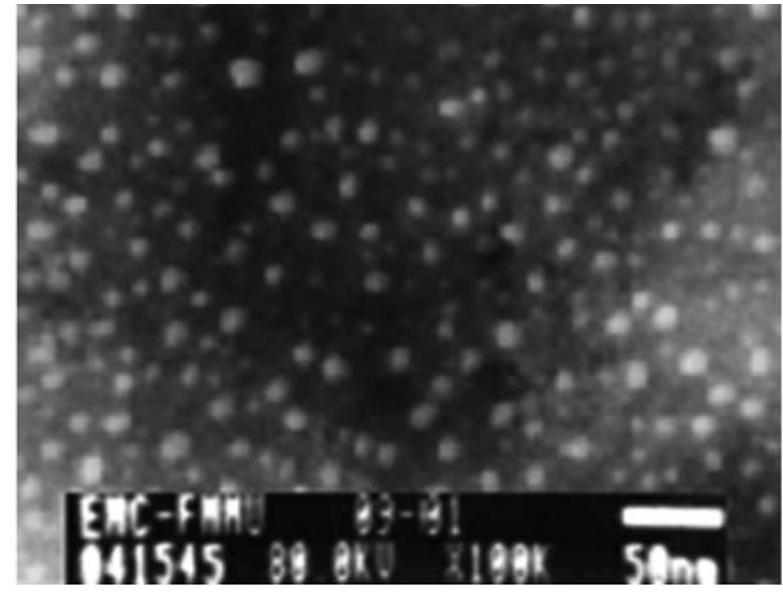

Figure 1. Nanoemulsion taken by transmission electro microscope (x100000). One drop of diluted nanovaccine (1:100) was dropped onto copper sieve, stained by $0.3 \%$ tungsten phosphate, and then observed under TEM when it dried. The vesicles in nanoemulsion showed approximately global shape with similar diameter, ranging from 15 to $25 \mathrm{~nm}$.

tively. The other mice were s.c. injected by $150 \mu 1 /$ mouse PBS or NE(-) as control. One week (D14) and 2 weeks (D21) later, these mice were immunized with the same regime as the first vaccination. Tumor volumes (length $\mathrm{x}$ width $^{2} \mathrm{x} \pi / 6$ ) were measured for each individual mouse and were plotted as the mean tumor volume of the group $( \pm$ SEM $)$ versus the number of days after tumor planted. Once tumors became palpable, measurements were taken twice a week. The mean survival time of mice was recorded.

Tumor challenge assay. Forty mice (10 per group) were vaccinated with $150 \mathrm{pmol} / 150 \mu \mathrm{l} / \mathrm{mouse} \mathrm{NE}(\mathrm{MHS})$ by po., i.v., s.c. or i.p. administration, and 20 mice (10 per group) were s.c. injected with $150 \mu \mathrm{l} /$ mouse PBS or NE(-) as control. One week and 2 weeks later, the immunization regime was repeated twice. On the 8 th day after the last immunization (D0), these mice were sc. challenged with B16-MAGE-1 tumor cells $\left(1 \times 10^{5}\right.$ cells/mouse, respectively) in the right legs. Once tumors became palpable, observations were taken twice a week. The ratio of tumor-free mice was recorded and Kaplan-Meier curves were generated.

Statistical analysis. One-way ANOVA was performed to determine differences of immune response among the various groups. Newman-Keuls tests were preformed as post-hoc analysis for one-way ANOVA. A P-value of $<0.05$ was considered significant.

\section{Results}

Characteristics of nanovaccine. NE(MHS) was a milky translucent homogeneous colloid. Transmission electron microscopy showed that the nanovaccine was spheric, and the average diameter was $20 \pm 5 \mathrm{~nm}$, as shown in Fig. 1. There was no delamination occurring following 6-month store at room temperature or $10 \mathrm{~min}$ centrifugation at $3000 \mathrm{rpm}$. The encapsulation efficiency was $87 \%$.

The MAGE-1-specific T-cell-mediated immune responses induced by vaccination with the NE(MHS) following different

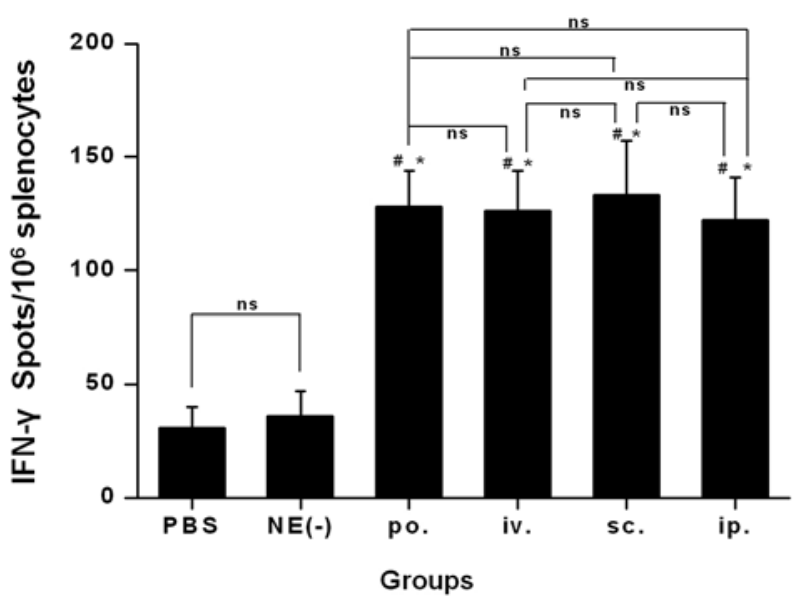

Figure 2. ELISpot assays of MAGE-1-specific T-cell precursors from the splenocytes of vaccinated mice via various immunization routes. C57BL/6 mice were vaccinated with $\mathrm{NE}(\mathrm{MHS})$ via op. i.v. s.c. or i.p., respectively. The control groups received PBS or NE(-) s.c. jnjection. Each mouse was immunized three times every 10 days. Mice were sacrificed on day 10 after the last immunization and splenocytes were isolated. The results are presented as mean \pm SEM. All analyses were done in duplicates. One-way ANOVA was performed for statistical analysis. ns denotes no significantly difference between the two groups. $\mathrm{P}<0.05$ was considered significant. "Denotes significantly different from PBS; *denotes significantly different from $\mathrm{NE}(-)$.

administration routes. $\mathrm{CD}^{+} \mathrm{CTLs}$ are one of the most crucial components among antitumor effectors (7). To determine the MAGE-1-specific CD8 ${ }^{+} \mathrm{T}$-cell precursor frequencies generated by NE(MHS), ELISpot and cytotoxicity assays were performed. ELISpot is a sensitive functional assay used to measure IFN- $\gamma$ production at the single cell level. As shown in Fig. 2, the numbers of spot-forming T-cell precursors specific for MAGE-1 in the splenocytes from mice vaccinated with $\mathrm{NE}(\mathrm{MHS})$ via different routes were all significantly more than that from mice with PBS or NE(-). Moreover, there were no statistical difference between the 4 different administration groups.

We also performed cytotoxicity assays to determine the MAGE-1-specific lysis of MAGE-1-expressing cells by CTLs induced by vaccination with NE(MHS) via 4 different routes. As shown in Fig. 3, there were no significant differences of the MAGE-1-specific lysis of CTLs between any 2 of po., i.v., s.c. and i.p. administration groups, and anyone of them was significantly higher than that of s.c. injection with NE(-) or PBS. The results were consistent with the data from ELISpost.

The treatment effect of $N E(M H S)$ vaccination following different administration routes. Sixty C57BL/6 mice were s.c. challenged with B16-MAGE-1 tumor cells in the right legs. One week later, tumor masses could be touched in $88.69 \%$ mice and all mice were randomly divided into 6 groups: po., i.v., s.c. and i.p. group, mice vaccinated with NE(MHS) by po., i.v., s.c. or i.p. route respectively, and PBS or NE(-) s.c. injection group. The same regime was repeated twice, 1 week (D14) and 2 weeks (D21) later. As shown in Fig. 4, from 14 days after the B16-MAGE-1 tumor challenge, vaccination with MHS nanovaccine significantly delayed tumor growth in B16-MAGE-1 tumor model compared with 


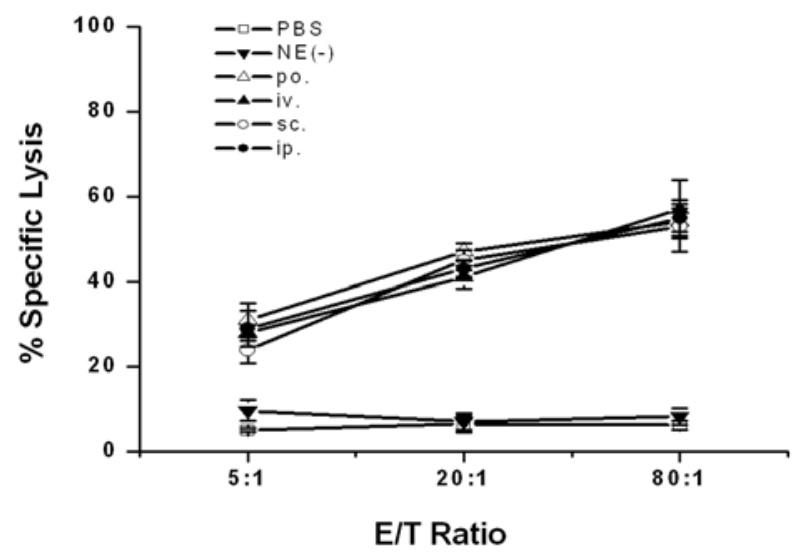

Figure 3. MAGE-1-specific lysis against B16-MAGE-1 cells by CTLs induced by vaccination with NE(MHS) via various immunization routes. Mice were vaccinated as described in the Fig. 2. The splenocytes of mice were harvested and restimulated with irradiated B16-MAGE- 1 cell. The percentage of specific lysis of CTLs on B16-MAGE-1 target cells was determined by a cytotoxicity assays. Percentage of MAGE-1-specific lysis was calculated by subtracting the percentage lysis of CTLs on B16 from that on B16-MAGE-1 target cells. Data shown represent average results obtained from six mice \pm SEM. All analyses were done in duplicates. The MAGE-1-specific lyses of CTLs from mice vaccinated with MHS nanoemulsion were same in the 4 different administration groups and they were all higher than that from mice injected with PBS or NE(-).

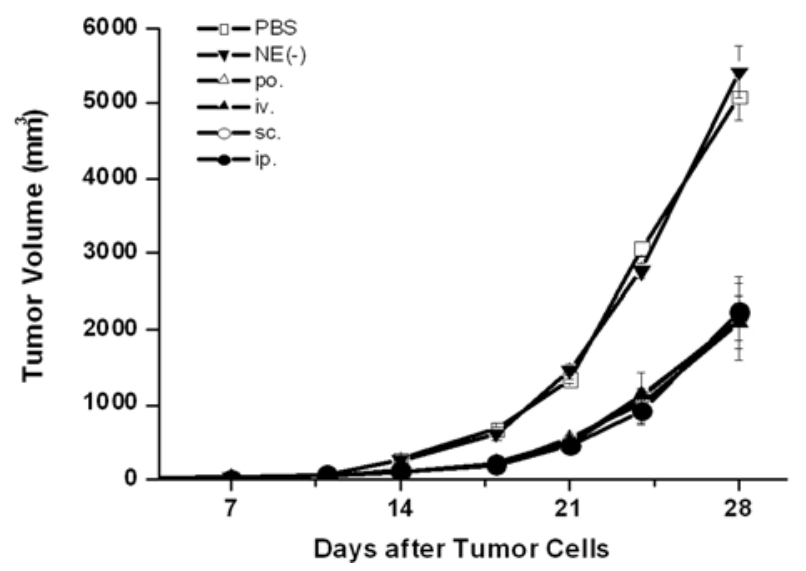

Figure 4. The immunotherapy effect of challenged B16-MAGE-1 melanoma with the tumor vaccine via different immune routes ( $\mathrm{n}=10$ mice/group). Group of mice were s.c. inoculated with B16-MAGE-1 tumor cells (1x10 cells/ mouse, respectively). Mice were vaccinated with $\mathrm{NE}(\mathrm{MHS})$ via 4 routes, or injected with PBS or NE(-) as control on day 7, 14 and 21. Data presented are mean \pm SEM. The tumor volumes on 7, 11, 14, 17, 21, 24 and 28 days after tumor cells were not significantly different in 4 groups via different administration routes. The tumor masses formed in control mice were statistically bigger than that of the $4 \mathrm{NE}(\mathrm{MHS})$ groups $(\mathrm{P}<0.01)$.

injection with PBS or NE(-), and there was no statistical difference in the 4 different administration groups at any observation point. At the same time, the survival times of mice were counted. As shown in Table I, compared with PBS and NE(-) groups, despite the fact that the administration routes were different, the survival times in the $4 \mathrm{NE}$ (MHS) groups were all significantly prolonged $(\mathrm{P}<0.001)$. However, the mean survival data of mice vaccined with $\mathrm{NE}(\mathrm{MHS})$ via 4 different routes were not statistically different $(\mathrm{P}>0.05)$.
Table I. The average survival time of mice treated with NE(MHS) via different immune routes.

\begin{tabular}{lc}
\hline Groups & Mean survival days (d) \\
\hline PBS & $24.80 \pm 6.37$ \\
NE(-) & $25.70 \pm 6.31^{\mathrm{ns}}$ \\
po. & $42.30 \pm 7.36^{\mathrm{a}-\mathrm{c}}$ \\
i.v. & $41.20 \pm 12.05^{\mathrm{a}-\mathrm{c}}$ \\
s.c. & $41.50 \pm 9.25^{\mathrm{a}-\mathrm{c}}$ \\
i.p. & $43.10 \pm 8.02^{\mathrm{a}-\mathrm{c}}$ \\
\hline
\end{tabular}

Vaccination with MHS nanoemulsion significantly prolonged the survival time compared with vaccination with PBS or NE(-). There were no significant differences in the mice of the 4 groups by statistical analysis in any 2 groups $(\mathrm{P}>0.05)$. ${ }^{\mathrm{a} D e n o t e s}$ no significantly difference between the any 2 of 4 different vaccination routes groups. ns, no significant difference from PBS. $\mathrm{P}<0.05$ was considered significant. ${ }^{b}$ significantly different from PBS. ${ }^{\mathrm{c}}$ significantly different from NE(-).

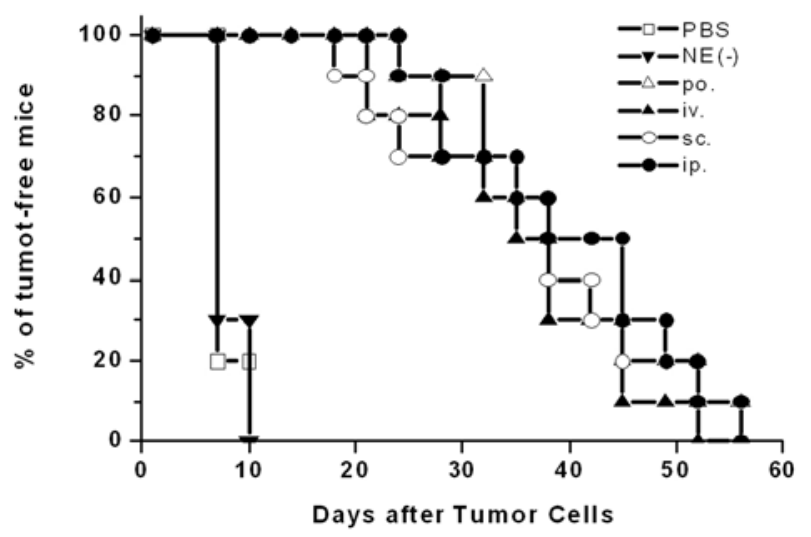

Figure 5. The preventive effect of challenged B16-MAGE-1 melanoma with the tumor vaccine via the different immune routes ( $n=10$ mice/group). The tumor-free rates of C57BL/6 mice inoculated with $\mathrm{NE}(\mathrm{MHS})$ vaccine via po., i.v., s.c. or i.p. route showed no statistical difference at anytime of observation after tumor cells. Compared with the mice s.c. injected with PBS or NE(-), the tumor occurrence time was significantly suspended in the mice vaccinated with MHS nanovaccine.

The protection effect of $N E(M H S)$ vaccination following different administration routes. To test the protection effect of NE(MHS) administrated via different routes, in vivo tumor challenge experiments were performed. As shown in Fig. 5, the tumor-free mice was recorded as the percentage of mice surviving from tumor after the tumor challenge and the percent of the tumor-free mice on the observation points in 4 groups showed no significant difference. In contrast, all mice in PBS and NE(-) group had a tumor by day 10 after B16-MAGE-1 tumor challenge, and none of NE(MHS) immunized mice had one 18 days after tumor challenge. So, the tumor occurrence time was significantly suspended in the mice vaccinated with MHS nanovaccine. 


\section{Discussion}

The goal of this study was to investigate the antitumor immune responses induced by nanoemulsion-encapsulated MAGE-1HSP70 and SEA as NE(MHS) vaccine following different administration routes and try to find new and effective immune routes. In this study, we showed that compared with PBS and NE(-) groups, the antitumor immune responses against MAGE-1 could be elicited by vaccination with MHS nanoemulsion. More importantly, vaccine immuni-zation through different routes did not produce significant difference on antitumor immune effects. Concerning nanoemulsion as a vaccinal approach enhancing the immune responses against antigens encapsulated in it has been proven in our previous study (5). The present novel and interesting finding is that the protein nanovaccine immunizing via po. is feasible.

Parenteral drug administration especially in chronic conditions is not well accepted by patients and may lead to issues with compliance. Consequently, the oral delivery of labile drugs is the focus of growing attention, particularly as many of the new therapeutic agents in development are hydrophilic drugs such as protein/peptide or oligonucleotides. However, numerous drugs especially protein/peptide vaccine remain poorly available when administered by this route. Among other reasons, this can be due to: i) low mucosal bioadhesion for the drug, ii) permeability restricted to a region of the gastrointestinal tract, iii) low or very low solubility of the compound which results in low dissolution rate in the mucosal fluids and elimination of a fraction of the drug from the alimentary canal prior to absorption, iv) lack of stability in the gastrointestinal environment, resulting in a degradation of the compound prior to its absorption (e.g. peptides, proteins). In order to circumvent some of these problems, it has been proposed to associate drugs with nanoparticulate systems (or small particles in the micrometre size range). Different oral administration experiments on animals have been reported, which have helped to improve the pharmacokinetics of several drugs (8-19), suggesting a high potential of nanoparticles as peroral drug delivery system (20). It has been reported that insulin incorporated into nanocapsules of poly(isobutyl cyanoacrylate) is unlikely to be degraded by gastrointestinal tract enzymes and therefore decreased glycemia by $50 \%$ in diabetic rats, which would otherwise be achieved through traditional oral administration $(13,14)$. It is demonstrated that the nanocapsules can well protect peptides from degradation by digestive enzymes (13).

Nanometer-sized drug delivery system has gained attention as a delivery formulation for peptide/protein and DNA drugs because it can protect labile drugs, increase drug solubility and bioavailability, control drug release and improve the bioadhesion and biopermesability when via po. administration (20). Nanoemulsion is a system of water, oil and amphiphile, which is a single optically isotropic and thermodynamically stable liquid solution smaller (diameter is 1-100 nm) but more efficient. In particular, nanoemulsion has become an important choice of protein/ peptide antigen drug delivery system because of its long circulation time and propensity to be phagocytosed more efficiently by antigen presenting cells to induce immune response $(21,22)$. Nanoemulsion of $100 \mathrm{~nm}$ diameter can be phagocytosed by reticulo-endothelial system from the blood, and that of $50 \mathrm{~nm}$ diameter or less can easily cross hepatic endothelium, reach spleen and bone marrow through lymphatics, and even reach the tumor, therefore, it is good for i.v. i.a. i.p. or po. (21-23). In this study, MAGE1-HSP70 fusion protein and SEA were coencapsulated in nanoemulsion as NE(MHS), of which the average diameter was $20 \pm 5 \mathrm{~nm}$. In addition, the immune responses induced by $\mathrm{NE}(\mathrm{MHS})$ via po., i.v., s.c. or i.p. route administration were approximately similar in C57BL/6 mice. The results suggest that nanoemulsion as a drug delivery of protein tumor vaccine makes the oral administration possible. The conceivable reasons are: i) nanoemulsion can protect hydrophilic drugs such as protein vaccine successfully incorporated into the dispersed aqueous phase of w/o microemulsion droplets where they are afforded some protection from enzymatic degradation when administered orally (24). In addition, the presence of surfactant and in some cases cosurfactant, for example medium chain diglycerides in many cases serves to increase membrane permeability thereby increasing drug uptake (24-30). ii) When nanoemulsion is administered orally, lymphatic uptake (31) by the M cells of the Peyer's patches appears to be a major site of translocation (32). Subsequent passage of nanoemulsion into mesenteric lymph nodes seems to be attributable to an uptake by macrophages (33). It was the base of nanoemulsion facilitating APC uptake of tumor antigens. Although the mechanisms for undiminished immune response to MAGE-1 induced by NE(MHS) oral administration in this research was not entirely clear, nanoemulsion as a carrier endowed the tumor vaccine new uses and merits.

In summary, our results indicated that peroral administration of nanoemulsion-encapsulated MHS complex protein vaccine could induce approximately similar antitumor immune responses to that via i.v., s.c. or i.p. routes, suggesting nanoemulsion is a promising carrier of MHS tumor vaccine and may have a broad application in cancer therapy.

\section{Acknowledgements}

This study was supported by grants from China National Natural Science Foundation (No.30271464, No.30700994 and No.30801357) and the Science and Technology Project of Shaanxi Province, China [No.2007K09-09(16) and No.2007C202].

\section{References}

1. Sudo T, Kuramoto T, Komiya S, et al: Expression of MAGE genes in osteosarcoma. J Orthop Res 15: 128-132, 1997.

2. Ma JH, Sui YF, Ye J, et al: Heat shock protein 70/MAGE-3 fusion protein vaccine can enhance cellular and humoral immune responses to MAGE-3 in vivo. Cancer Immunol Immunother 54: 907-914, 2005.

3. Torres BA, Kominsky SL, Perrin GQ, et al: Superantigens: the good, the bad, and the ugly. Exp Biol Med 226: 164-176, 2001.

4. Fleischer B and Schrezenmeier H: T cell stimulation by staphylococcal enterotoxins. Clonally variable response and requirement for major histocompatibility complex class II molecules on accessory or target cells. J Exp Med 167: 1697-1707, 1988. 
5. Ge W, Sui YF, Wu DC, et al: MAGE-1/Heat shock protein 70/ MAGE-3 fusion protein vaccine in nanoemulsion enhances cellular and humoral immune responses to MAGE-1 or MAGE-3 in vivo. Cancer Immunol Immunother 55: 841-849, 2006.

6. Ye J, Chen GS, Song HP, et al: Heat shock protein 70/MAGE-1 tumor vaccine can enhance the potency of MAGE-1-specific cellular immune responses in vivo. Cancer Immunol Immunother 53: $825-834,2004$

7. Melief CJ and Kast WM: T-cell immunotherapy of tumors by adoptive transfer of cytotoxic $\mathrm{T}$ lymphocytes and by vaccination with minimal essential epitopes. Immunol Rev 145: 167-177, 1995.

8. Alpar HO, Field WN, Hayes K and Lewis DA: A possible use of orally administered microspheres in the treatment of inflammation. J Pharm Pharmacol 41: 50-53, 1989.

9. Ammoury N, Fessi H, Devissaguet JP, et al: Jejunal absorption, pharmacological activity, and pharmacokinetic evaluation of indomethacin-loaded poly (D, L-lactide) and poly(isobutylcyanoacrylate) nanocapsules in rats. Pharm Res 8: 101-105, 1991.

10. Beck PH, Kreuter J, Müller WEG and Schatton W: Improved peroral delivery of avarol with polyalkylcyanoacrylate nanoparticles. Eur J Pharm Biopharm 40: 134-137, 1994.

11. Bonduelle S, Carrier M, Pimienta C, et al: Tissue concentration of nanoencapsulated radiolabelled cyclosporin following peroral delivery in mice or ophthalmic applications in rabbits. Eur $\mathbf{J}$ Pharm Biopharm 42: 313-319, 1996.

12. Damgé C, Aprahamian M, Balboni G, et al: Polyalkylcyanoacrylate nanocapsules increase the intestinal absorption of a lipophilic drug. Int J Pharm 36: 121-125, 1987

13. Damgé C, Michel C, Aprahamian M, et al: Nanocapsules as carriers for oral peptide delivery. J Control Release 13: 233-239, 1990.

14. Damgé C, Michel C, Aprahamian M and Couvreur P: New approach for oral administration of insulin with polyalkylcyanoacrylate nanocapsules as drug carrier. Diabetes 37: 246-251, 1988.

15. Damgé C, Vonderscher J, Marbach P and Pinget M: Poly(alkyl cyanoacrylate) nanocapsules as a delivery system in the rat for octreotide, a long-acting somatostatin analogue. J Pharm Pharmacol 49: 949-954, 1997.

16. Hubert B, Atkinson J, Guerret M, et al: The preparation and acute antihypertensive effects of a nanocapsular form of darodipine, a dihydropyridine calcium entry blocker. Pharm Res 8: 734-738, 1991.

17. Maincent P, Le Verge R, Sado P, et al: Deposition kinetics and oral bioavailability of vincamine-loaded polyalkyl cyanoacrylate nanoparticles. J Pharm Sci 75: 955-958, 1986.

18. Mathiowitz E, Jacob JS, Jong YS, et al: Biologically erodible microspheres as potential oral drug delivery systems. Nature 386: 410-414, 1997.
19. Ottenbrite R, Zhao R and Milstein S: A new oral microsphere drug delivery system. Macromol Symp 101: 379-387, 1996

20. Ponchel G and Irache JM: Specific and non-specific bioadhesive particulate systems for oral delivery to the gastrointestinal tract. Adv Drug Deliv Rev 34: 191-219, 1998.

21. Niidome $\mathrm{T}$ and Huang L: Gene therapy progress and prospects: non-viral vectors. Gene Ther 9: 1647-1652, 2002.

22. Vinogradov SV, Bronich TK and Kabanov AV: Nanosized cationic hydrogels for drug delivery: Preparation, properties and interactions with cells. Adv Drug Deliv Rev 54: 135-147, 2002.

23. Jores K, Mehnert W, Drechsler M, et al: Investigations on the structure of solid lipid nanoparticles (SLN) and oil-loaded solid lipid nanoparticles by photon correlation spectroscopy, fieldflow fractionation and transmission electron microscopy. J Control Release 95: 217-227, 2004.

24. Sarciaux JM, Acar L and Sado PA: Using microemulsion formulations for drug delivery of therapeutic peptides. Int $\mathbf{J}$ Pharm 120: 127-136, 1995.

25. Constantinides PP, Lancaster CM, Marcello J, et al: Enhanced intestinal absorption of an RGD peptide from water-in-oil microemulsions of different composition and particle size. J Control Rel 34: 109-116, 1995.

26. Constantinides P: Lipid microemulsions for improving drug dissolution and oral absorption: physical and biopharmaceutical aspects. Pharm Res 12: 1561-1572, 1995.

27. Constantinides PP, Scalart JP, Lancaster S, et al: Formulation and intestinal absorption enhancement evaluation of water-in-oil microemulsions incorporating medium-chain glycerides. Pharm Res 11: 1385-1390, 1994.

28. Pouton CW: Formulation of self-emulsifying drug delivery systems. Adv Drug Deliv Rev 25: 47-58, 1997.

29. Swenson EC and Curatolo WJ: Intestinal permeability enhancement for proteins, peptides and other polar drugs: mechanisms and potential toxicity. Adv Drug Deliv Rev 8: 39-92, 1992.

30. Swenson ES, Milisen WB and Curatolo W: Intestinal permeability enhancement: efficacy, acute local toxicity and reversibility. Pharm Res 11: 1132-1142, 1994.

31. Pappo J and Ermak TH: Uptake and translocation of fluorescent latex particles by rabbit Peyer's patch follicle epithelium: a quantitative model for $\mathrm{M}$ cell uptake. Clin Exp Immunol 76: 144-148, 1989.

32. O'Hagan DT: Intestinal translocation of particulates - implications for drug and antigen delivery. Adv Drug Deliv Rev 5: 265-285, 1990.

33. Devissaguet JP, Fessi H, Ammoury N and Barratt G: Colloidal drug delivery systems for gastrointestinal application. In: Drug Targeting and Delivery-Concepts in Dosage Form Design. Junginger HE (ed). Ellis Horwood, New York, pp71-91, 1992. 